\title{
A!
}

This is an electronic reprint of the original article.

This reprint may differ from the original in pagination and typographic detail.

Davis, Hilary; Wilde, Danielle; Altarriba Bertran, Ferran; Dolejsova, Marketa

\section{Fantastic(e)ating Food Futures}

Published in:

DIS 2020 Companion - Companion Publication of the 2020 ACM Designing Interactive Systems Conference

DOI:

https://doi.org/10.1145/3393914.3395906

Published: 06/07/2020

Document Version

Peer reviewed version

Please cite the original version:

Davis, H., Wilde, D., Altarriba Bertran, F., \& Dolejsova, M. (2020). Fantastic(e)ating Food Futures: Reimagining Human Food Interaction. In DIS 2020 Companion - Companion Publication of the 2020 ACM Designing Interactive Systems Conference (pp. 377-380). ACM. https://doi.org/10.1145/3393914.3395906

This material is protected by copyright and other intellectual property rights, and duplication or sale of all or part of any of the repository collections is not permitted, except that material may be duplicated by you for your research use or educational purposes in electronic or print form. You must obtain permission for any other use. Electronic or print copies may not be offered, whether for sale or otherwise to anyone who is not an authorised user. 


\section{Fantastic(e)ating Food Futures: Reimagining Human Food Interactions}

\section{Hilary Davis}

Swinburne University of

Technology \& La Trobe University,

Melbourne, Australia

hdavis@swin.edu.au

\section{Danielle Wilde}

University of Southern Denmark

Kolding, DK

wilde@sdu.dk

\section{Ferran Altarriba Bertran}

Social Emotional Technology

Lab - UC Santa Cruz

Santa Cruz, CA, USA

ferranaltarriba@gmail.com

\section{Markéta Dolejšová}

Aalto University, Espoo, Finland

marketa.dolejsova@gmail.com

Permission to make digital or hard copies of part or all of this work for personal or classroom use is granted without fee provided that copies are not made or distributed for profit or commercial advantage and that copies bear this notice and the full citation on the first page. Copyrights for thirdparty components of this work must be honored. For all other uses, contact the Owner/Author.

DIS '20 Companion, July 6-10, 2020, Eindhoven, Netherlands (C) 2020 Copyright is held by the owner/author(s).

ACM ISBN 978-1-4503-7987-8/20/07.

https://doi.org/10.1145/3393914.3395906

\begin{abstract}
According to EAT-Lancet: Food is the single strongest lever to optimize human health and environmental sustainability on Earth. However, current food practices are threatening both people and planet. Digital food technologies offer potential for efficient food lifestyles but they present limited opportunity for imagining 'fantastic' food futures. In a bid to extend Human-Food Interaction (HFI) research, this workshop will investigate how to fantasticate nourishing ways to technologically support food practices. Through scenario building, mystery food-tech boxes, Food Tarot cards, and walk-shop, we will feed our senses, fuel imaginations, seek HFI design possibilities and reflect on their potential to nurture healthy, sustainable human-food relationships. The workshop is organized as part of a two-day program titled Experimental Food Design for Sustainable Futures inviting diverse participants interested in contributing toward sustainable socio-ecological transformations.
\end{abstract}

\section{Author Keywords}

Human-food interaction; HFI; digital food cultures; food design; food futures; mystery boxes, fantastic 


\section{Workshop Schedule \\ (July 6, 2020)}

9-9.30am: Introductions \& scene setting

\section{9:30-11.30pm:}

Presentations and discussion 11:30-12.30pm: Food Tarot cards and future scenarios

12:30-2.30pm: Lunch and walk-shop

2.30-4pm: Food-tech

mystery boxes

4-5:00pm: Fantastic Food

Futures Cookbook

5-5.30pm: Conclusion and

plans for the future

The workshop is part of a 2 day program Experimental

Food Design for Sustainable

Futures. Each day focuses on a distinct theme, using food

as an accessible starting

point from which to explore

and articulate values,

concerns, desires, and

imaginaries associated with

societal transformation

towards sustainable futures.

DIS attendees have the

option to sign up for both

workshop days or for one day

only; two-day participation is

encouraged. Details about

the 2-day program:

https://experimentalfooddesi

gn.wordpress.com/

\section{CSS Concepts}

- Human-centered computing HCI design and evaluation methods - HCI theory, concepts and models

\section{Introduction}

The call to action of the 2019 EAT-Lancet report [7] is unequivocal: humans must urgently develop more nourishing ways of producing and consuming food. Technology increasingly permeates food cultures; we believe Human Computer Interaction (HCI) and DIS communities have an important role to play in shaping necessary changes in food practices and processes. While claiming that 'food is the new internet' [4], some food-tech proponents portray technology as a means to revolutionise food systems. In contrast, critics are concerned that digital food efforts are leaning towards technological solutionism-the belief that technology design can fully solve complex societal problems [3]. We see a lack of critical HFI research that supports imagination of future food practices that can nourish both people and the planet [1]. Our response to this challenge is to push the boundaries of HFI by including more imaginative, fanciful or fantastical approaches.

\section{Motivation and Goals}

This workshop seeks to move beyond the possibilities and constraints of new technologies by fantasticating (making or rendering fantastic or fanciful) foodtechnology futures. The goal is to imaginatively explore ways to intertwine human and planetary flourishing through food-tech innovation. To 'fantasticate HFI', we will explore novel food-tech devices, both existing and emerging, and enjoy thought-provoking workshop activities informed by participant-related contexts. We will approach food-technology practices as contested areas navigated by multiple stakeholders including human and non-human actors from the natural ecology and the technologies themselves. The aim is to interrogate: The advantages and challenges that digital food technology brings to everyday-life contexts. How to incorporate perspectives that embrace the morethan-human. How to leverage creative and imaginative design approaches to scaffold the development of fantastical but sustainable, just food-tech cultures? How to design technologies that extend beyond deterministic approaches to support imaginations of playful and fantastical food futures.

\section{Structure and Activities}

The workshop is organized as part of a two-day program titled Experimental Food Design for Sustainable Futures. The two-day program focuses on experimenting with food as a bio-design material and socio-culturally potent, aesthetically rich starting point from which to critically reflect on social and ecological uncertainties. This workshop (day 1) uses experimenta food design co-creation to examine interdependencies between food and technology, and 'fantasticate' future food-tech practices navigated by diverse human and non-human stakeholders. Day 2 of the workshop experiments with food to explore climate change uncertainties and speculate how more-than-human perspectives might be included to support sustainability. The two workshop days are thematically intertwined and have been carefully designed to be complementary.

Drawing on our prior DIS workshops $[2,5]$, we aim to help nurture existing research into everyday foodtechnology cultures and develop a strong community of HFI scholars. While ensuring inclusion of fantastical elements such as food crafting, play, foraging, future 


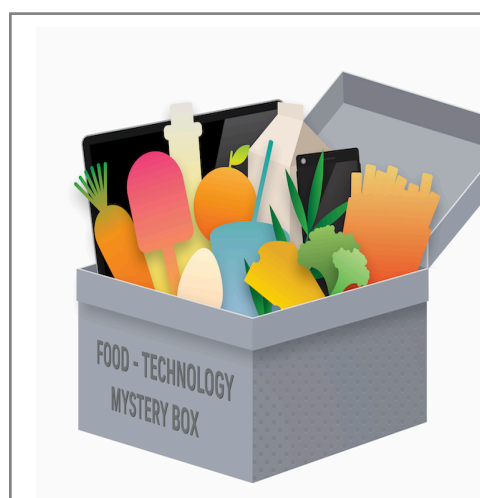

Figure 1: Food-tech mystery boxes will be used to reimagine future food interactions.

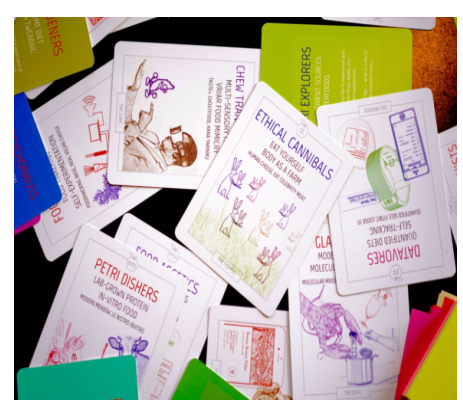

Figure 2: Food Tarot cards will be used for small-group scenario building. speculations, and food-tech mystery boxes (figure 1) reminiscent of 'magic box' cultural probes [6] we will leverage the cultural and material richness of food for creative albeit critical thinking. Our workshop contributes to burgeoning $\mathrm{HFI}$ research and related $\mathrm{HCI}$ inquiries into data-driven health and 'green' lifestyles, while reimagining alternate food futures. We invite a wide range of multi-disciplinary and intersectional contributions from food-oriented researchers, designers, artists, scientists, DIY biohackers, and other practitioners concerned with food-technology issues. We welcome diverse contributions, including: ethnographic and participatory research in citizen food communities, experimental food design research, foodoriented performance art and theater play, digital food fabrication and DIY food bio-hacking. By fantasticating human-food interactions, we aim to imagine future HFI frameworks. The organisers will shepherd workshop activities to ensure inclusive, invigorating exchange.

\section{Workshop Themes}

The workshop reflects on the implications of using digital technology for everyday food practices by reimagining or fantasticating food futures. The themes of the workshop cover (but are not limited to):

\section{Personal implications}

How can personal digital technology be reimagined to forge better HFI experiences? How can we use or repurpose existing food technologies to reimagine more fulfilling personal experiences? How might we design to best support the food-related health, wellbeing, and resilience of individuals and communities?

2. Socio-cultural implications: How do technologies affect traditional food practices and culinary techniques, and which of these are valued? How can we reimagine and thereby support improved social food connectivity and commensality? What might food communities of the future resemble? What roles might traditional food knowledge (e.g., foraging) play in the future?

\section{Environmental implications}

To what extent can digital technologies support sustainable food practices? What are the opportunities of digital technology in advancing users' ecological connectedness? How can we design for playful, creative, and fantastical but also critical user engagement with sustainable food practices?

\section{Policy implications}

What kind of data will be produced and shared via future digital food technologies, by whom, and to what ends? Who will be excluded from future digital food practices? How can we design to support a safe exchange of personal food-related data?

\section{Workshop Structure \& Activities:}

This full-day workshop will involve short participant presentations followed by group discussions, playful prototyping, a curated walk-shop and scenario-building via food-tech mystery boxes and Food Tarot cards [8].

Introduction (9-9.30 am): We introduce the themes, agenda and activities for the day. Participants introduce a boundary object to open a reflective space around an aspect of food technology.

Presentations (9.30-11.30 am): Participants deliver brief presentations, elaborating their boundary object and food technology interests, followed by a short Q\&A, with sufficient time for reflective feedback. We then 
hold a group discussion of how the presented ideas and artifacts might inform fantastical HFI food futures.

Food Tarot Cards (11.30am-12.30pm): Food Tarot Card [8] guided scenario-building and prototyping will outline existing and anticipated speculative food-tech practices. The Food Tarot deck is designed to raise questions about current and near-future food-tech issues, and provoke players to fantasticate: What, where and how will we eat in the near future?

Lunch and walk-shop (12.30-2.30pm): The walkshop will include foraging for local food-related artefacts e.g. flowers, herbs, fruits, used to seed the afternoon activities. Walk-shop participants will use mobile phones to document experiences. Foraged artifacts and documentation will inform future publications, digital story compilation and online digital cookbook.

Food-Tech mystery boxes (2.30-4pm): Using foodtech mystery boxes, we reimagine future food interactions. The mystery boxes contain playful, participatory food and tech artifacts designed to prompt thinking through imagining, moving, making and doing. The props are based on 'magic box' cultural probes [6] and take inspiration from real and imagined food exchanges, activities and mealtimes.

Reimagining Fantastical Food Futures (4-5pm):

Groups will showcase scenarios and share critical reflections on their design approaches. The reflections, scenarios, and digital story will inform an online recipe book of fantastical HFI ideas and design prototypes shared on the workshop website:

experimentalfooddesign.wordpress.com
Conclusion and plans for the future (5-5.30pm): Participants may contribute to our International Journal of Food Design special issue, confirmed for early 2021.

\section{References}

[1] Ferran Altarriba Bertran, Samvid Jhaveri, Rosa Lutz, Katherine Isbister and Danielle Wilde. 2019. Making Sense of Human-Food Interaction. In $\mathrm{CHI}$ Proceedings May 4-9, 2019, Glasgow, Scotland UK. ACM, New York, NY, USA.

[2] Markéta Dolejšová, Ferran Altarriba Bertran, Danielle Wilde, and Hilary Davis, 2019. Crafting and Tasting Issues in Everyday Human-Food Interactions. Designing Interactive Systems Conference 2019 Companion ACM, 361-364.

[3] Evgeny Morozov. 2013. To Save Everything Click Here: Technology, Solutionism and the Urge to Fix Problems That Don't Exist. Allen Lane Penguin.

[4] Kimbal Musk. (2016-2017). Food is the New Internet. Medium. Retrieved October 12, 2017 from http://medium.com/food-is-the-new-internet

[5] Erica Vannucci, Ferran Altarriba, Justin Marshall, and Danielle Wilde. 2018. Handmaking Food Ideals: Crafting the Design of Future Food-related Technologies. Designing Interactive Systems (DIS '18 Companion). ACM, New York, USA, 419-422.

[6] Frank Vetere, Hilary Davis, Martin Gibbs, Steve Howard. 2009. The magic box and collage: responding to the challenge of distributed intergenerational play, International Journal of Human-Computer Studies, Vol. 67, no. 2 (Feb 2009), pp. 165-178

[7] Willett, W. et al. Food in the Anthropocene: the EAT-Lancet Commission on healthy diets from sustainable food systems. The Lancet 393, 447492 (2019)

[8] Parlour of Food Futures. Retrieved January 10, 2020 from https://foodtarot.tech/ 